\title{
Quantitative inversion of sparse vegetation coverage in desertification area
}

\author{
Xuedong $\mathrm{Li}^{1,}$,, Xuya Zhang ${ }^{2, b}$, HongYan Zhang ${ }^{1, \mathrm{c}}$, Guang bin Yang $^{3, \mathrm{~d}, \star}$ \\ ${ }^{1}$ School of Geographical Science, Northeast Normal University, Jilin, 130024, China \\ ${ }^{2}$ College of Resource Environment and Tourism, Capital Normal University, Beijing, 100048, China \\ ${ }^{3}$ School of Geography and Environment Sciences, Guizhou normal university, Guizhou, 550001, \\ China \\ ali.xuedong.1988@163.com, 'lionhenryzxy@sina.com, ‘66487566@qq.com, dygbyln@163.com
}

Keywords:Desertification, Sparse vegetation, Quantitative remote sensing.

\begin{abstract}
Based on unmixing model and using the Thematic Mapper (TM) image as well as obtaining end-members by ground spectral measurements, quantitative retrieval of information on sparse vegetation coverage in oasis-desert transitional area in Minqin, Gansu was done. The results showed that a wide band of TM images can be used in extracting sparse vegetation coverage of arid regions. Three components were needed to build unmixing model and there includes light, vegetation - not light and vegetation - bare soil. And the unmixing model has a high correlation with measured vegetation coverage. Methods used to choose the end-members had certain influence on estimating the vegetation coverage. Monte Carlo method recorded 0.64 as coefficient of determination (R2) 3.8 as root mean square error (RMSE) and therefore was more accurate than the root mean square error minimization method.
\end{abstract}

\section{Introduction}

Arid region represents about one-third of the world's land area and has extremely fragile ecosystems. More and more land in arid regions is suffering from desertification. The main reasons are the irrational land use and climate change. Evaluation of vegetation coverage is considered to be the most effective desertification indicators [1,2]. Obtaining accurate vegetation coverage in arid areas is important for accurate assessment of desertification and environment protection.

Environment in deserts is special. The contribution of sparse vegetation in the spectral reflectance in each pixel is small. Pixels usually render characteristics of the soil. These make difficult to accurately estimate sparse vegetation in desertification area by remote sensing methods [3]. So far, unmixing method has been widely used in the estimation of vegetation coverage. The method extracts information in sub-pixel scale instead of pixel scale. Many researches showed that this method is much better than the normalized difference vegetation index method [4,5] use in estimating vegetation coverage. Although the components of end-members in each pixel are the same, with the impact of natural background, the same component between the pixels is different. Therefore, using fixed set of end-member to unmix will inevitably influence the estimation accuracy [6,7]. Asner achieved good results by using TM images as data source and linear unmixing model to estimate vegetation coverage in the Amazon basin forest [8,9]. However, the method has not been effectively verified in areas of sparse vegetation. Many researches rarely use wide-band TM data. Therefore, this study carried out on the Minqin area, used TM data as data source and unmixing method to extract information of weak vegetation in desertification areas.

\section{Materials and Methods}

\subsection{Preprocessing of remote sensing image}

Landsat 5 Thematic Mapper (TM) imagery of the Minqin's region was acquired on 252, 2013 (path 131, row 33). Geometric correction was done on the high resolution images with ground control point coordinates. Then atmospheric correction with the FLAASH module provided by ENVI was 
done. Spectral characteristics of the image were ensured to be consistent with the measured spectral characteristics of surface features.

\subsection{Spectra measurement}

The Field SpecPro FR spectrometer was used to measure spectra on the Field. The spectrometer was made by American analytical spectral device (ASD) company. Its viewing angle is 25 degree. Spectral range is from $350 \mathrm{~nm}$ to $2500 \mathrm{~nm}$. Sampling interval was $1.4 \mathrm{~nm}$ when spectra were between $350 \mathrm{~nm}$ and $1000 \mathrm{~nm}$ and $2 \mathrm{~nm}$ in the range of 1000-2500 nm. Measurements of spectra on the field and image acquisition were done at the same time, from 10:00 to 14:00. That day was a nice day with a perfect weather, no wind and clouds. Vertical observation was taken in spectral measurements. There was $40 \mathrm{~cm}$ from fiber tip to canopy or surface. Optimization of reference version was taken every five minutes during the spectrum measurement. After the measurement, 440 spectra including 178 different features were measured. These features included Haloxylon, white thorn, thorn bubble, surface crust, sand, dry white thorn, dry Haloxylon and so on. Meanwhile the locations and the corresponding landscape photos and fisheye photos were recorded

The measured ground spectra provide rich spectrum information, which helps to get knowledge on the feature, while the information redundancy caused by the increasing in bands gives difficulty with data processing [10-11]. Therefore, the extraction of spectral feature and data optimization become very important. In this study, according to PV, NPV, SOIL, we set up different measured ground spectral libraries to store the spectrum information measured on the field. And we resampled the obtained images in accordance with the spectral response function and got the feature classes in each image.

It is necessary to consider influence from topographic and atmospheric when unmixing the pixels by using the measured spectra [12]. In this research, we used standardized methods to decrease these effects. As shown in figure 1, after standardizing, the relative difference in reflectance intensity has been removed and surface reflectance characteristics have been better reflected.
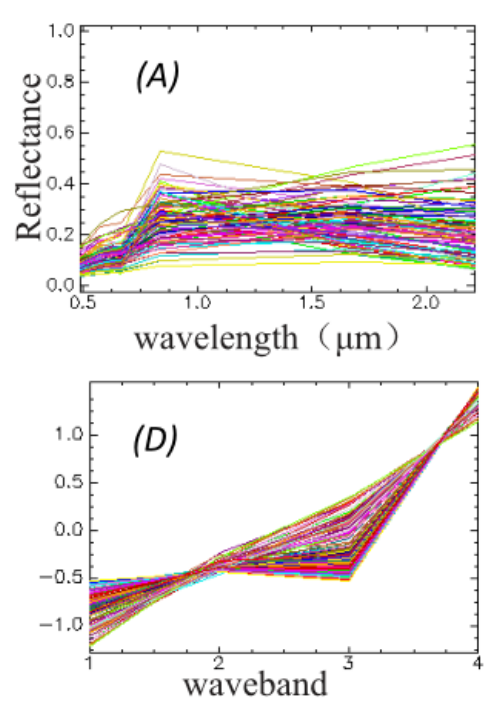
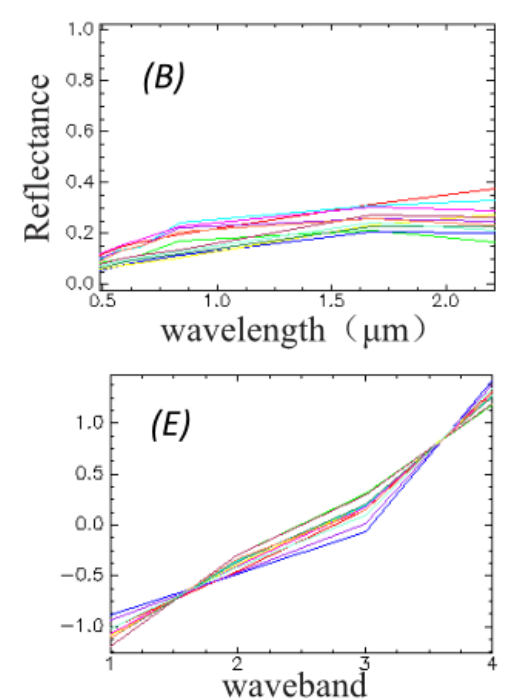
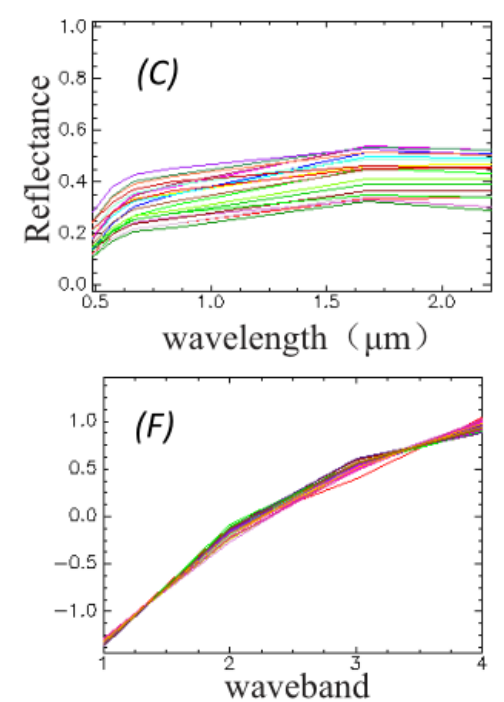

Figure 1 end-member spectral library (A, B, C show spectrum which is resampled according to the TM spectrum response function. $\mathrm{D}, \mathrm{E}, \mathrm{F}$ is the normalized spectrum, in which the $\mathrm{X}$-axis represents the band 3, 4, 5, and 7 of TM image. The $y$-axis is the reflectance after normalization, in which $\mathrm{A}$ and $\mathrm{D}$ are for PV; B and E are for NPV; and C and F are for SOIL)

\subsection{Field measurement of vegetation coverage}

Measurement of spectra on the Field and image acquisition was done at the same time. There was no significant precipitation, frost and impact on vegetation during the field survey. Considering spatial resolution ( $30 \mathrm{~m}$ ) of TM images, this study set the size of sample area to $25 \mathrm{~m} * 25 \mathrm{~m}$. Trimble handheld GPS was used to record coordinates of sampled area's center and corners and fish-eye camera was used to take pictures in an equidistant way. The camera was set perpendicular to the sample line. Vegetation coverage of sampled area was estimated according to the results from processing fisheye photographs with Caneye. 


\subsection{Inversion of surface coverage}

Spectral mixing model is divided into linear and nonlinear models. Non-linear model considers the multiple scattering in the end-elements. But the processing has revealed that there is no linear relationship between the influence of multiple scattering and the end-member proportion in pixels. However the linear model was relatively simple and physical sense was clear. Therefore the simple linear spectral mixture was assumed in this study and the formula is as follows:

$$
D_{N i}=\sum_{i=1}^{L} \sum_{j=1}^{P} m_{i j} \alpha_{j}+e_{i}(1)
$$

Where: $D_{N i}$ is the pixel reflectance in the $i$ band; $\mathrm{L}$ is the number of spectral bands; $\mathrm{p}$ is the number of end-member in the pixel; $m_{i j}$ is the reflectance for thejend-member in the pixel in theiband; $\alpha j$ is the proportion of jend-member in the pixels; eiis error for the $i$ band. To make LSMM provide accurate and reliable estimate of the vegetation cover, there must be two limitations: i) the sum of component of each end-member equals to 1. ii) component of each end-member of the elements is non-negative. LSMM with these two limitations is called whole limited LSMM.

\section{Result and Discussion}

\subsection{Extraction of vegetation coverage based on unmixing model}

In this study, unmixing the image of experimental area was based on linear unmixing model. The methods used for selecting end-member were: root mean square error minimization method (RMSE minimum algorithm) and Monte Carlo simulation algorithms. Based on measured data from sampled areas, the accuracy of vegetation coverage extraction was estimated. Coefficient of determination, root mean square error and the estimation accuracy were selected as indices to evaluate the model i.e. to compare the measured values with the estimated values. As illustrated on figure 2, Monte Carlo method had the highest precision showed by points basically distributed around the ' $\mathrm{Y}=\mathrm{X}$ ' reference line. The root mean square error recorded less than 4 , the estimation accuracy equal to 0.61 and the coefficient of determination is 0.82. For the RMSE minimization method, the RMSE was 5.13 and estimation accuracy equal to 0.54. Thus, the Monte Carlo simulation algorithms appeared more accurate than RMSE minimum method in estimating vegetation coverage. Therefore, it is suggested to use Monte Carlo simulation algorithms to select end-member and then unmixing model based on linear spectrum to extract information of weak vegetation in areas under desertification.
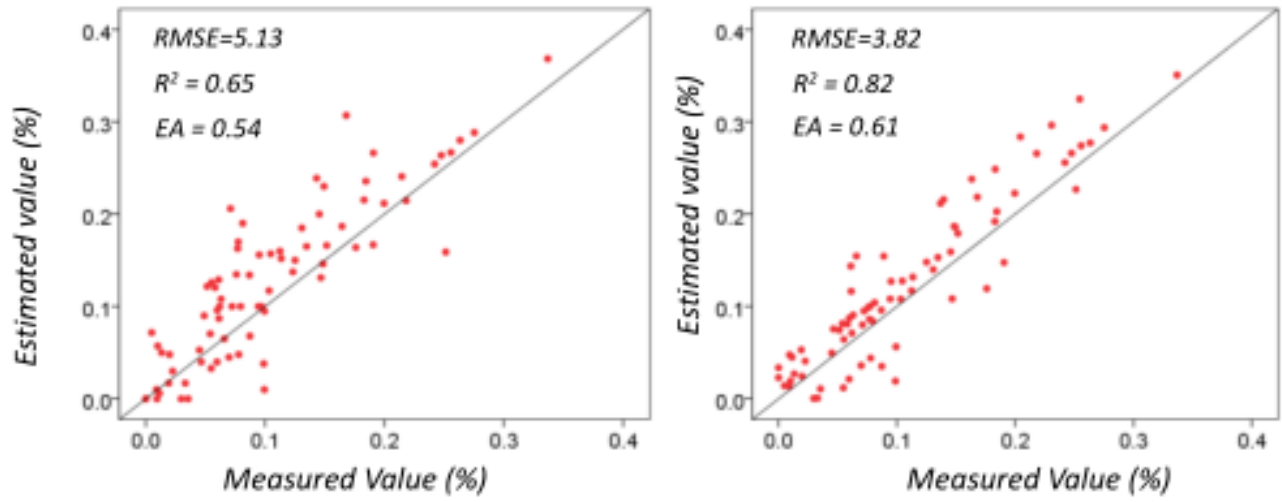

Figure 2 Scatterplot about estimates of weak vegetation in desert and value of measured vegetation coverage from two selected methods

\subsection{Sparse vegetation coverage of the study area}

The vegetation coverage of the study area resulting from Monte Carlo simulation algorithms and unmixing method is shown by Figure 3. From the perspective of spatial distribution, the vegetation coverage in the study area has been well represented. The lower portion of the study area is farmland and vegetation is relatively concentrated there. Then this area forms an obvious desert oasis where vegetation coverage is relatively large. The maximum of vegetation coverage is close to the peak value while desert has lower vegetation coverage. The vegetation coverage of most desert area was 
less than 30\%. Even in some regions, there was no vegetation at all. The low vegetation cover in the study area contributes to the expansion of desertification, which causes a serious threat to farmland area in desert oasis.
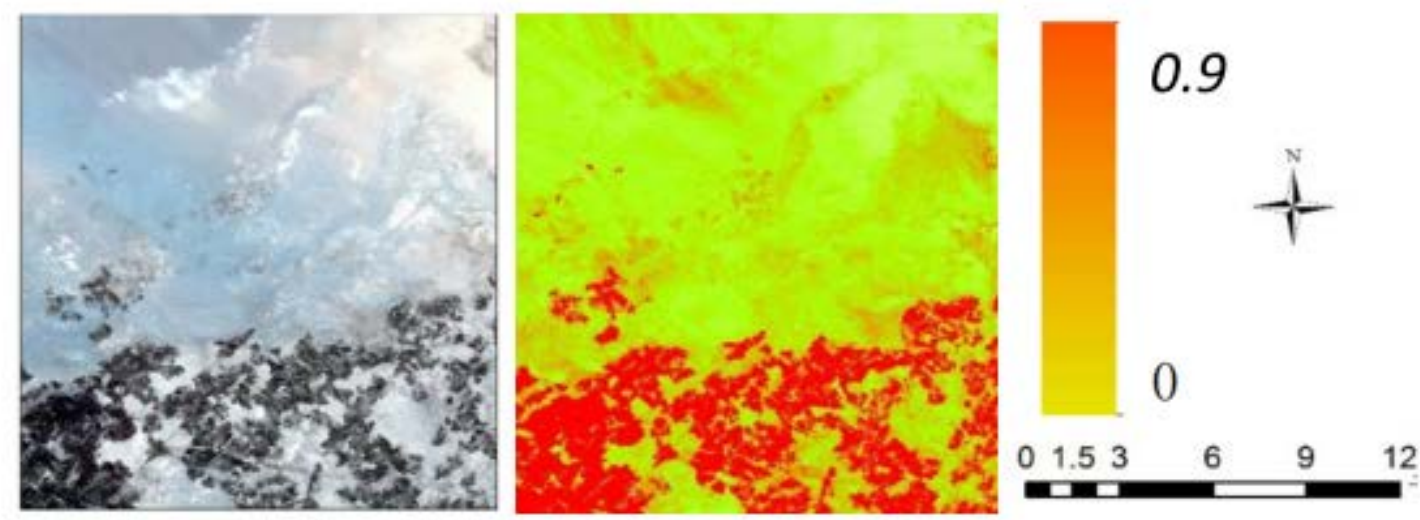

$K m$

Figure 3 vegetation coverage estimation results

\section{Summary}

In this study, TM image and measured spectra from field survey have been used to build ternary unmixing model in desertification areas and achieve the extraction of sparse vegetation coverage. The main conclusions were as follows:

1) It appeared feasible to extract weak vegetation Information in desertification areas using TM Images. Standardize treatment on image and the measured spectrum effectively reduced the interference of physical factors and improved accuracy of unmixing

2) The PV-NPV-SOIL ternary unmixing model can be effectively used for extracting information about weak vegetation in desertification areas. Monte Carlo simulation algorithms had higher accuracy than the RMSE method.

3) Vegetation coverage in study area was very low. This area showed obvious vegetation degradation. Farmland was relatively concentrated and formed an obvious desert oasis. Around the oasis, desertification put threats on desert oasis.

This study realized extraction of information on vegetation. But due to band limitation, between the NPV and SOIL, there are still some uncertainties which are worth exploring. Meanwhile because of the environment, the depths of the desert cannot be reached. These reasons described above result the field data and survey of sample areas are concentrated. There for The data cannot meet the estimation of larger area.

\section{Acknowledgement}

This work is supported by Nation Science Foundation of China (41361091), science and technology Foundation of GuiZhouprovince (LKS[2012]26), major special projects Foundation of GuiZhou province ([2012]6007),the corresponding author is Dr. Guangbin Yang, Guizhou normal university, dygbyln@163.com

\section{Reference}

[1]Liao Chun-hua, ZHANG Xian-feng, LIU Yu. Remote sensing retrieval of vegetation coverage in arid areas based on multiple endmember spectral unmixing. Chinese Journal of Applied Ecology, 2012, 23(12): 3243-3249

[2]Zhang Y-X, Li X-B, Chen Y-H. Overview of field and multi-scale remote sensing measurement app roaches to grassland vegetation coverage. Advances in Earth Science.2003, 18 (1) : 85- 93 
[3]Arsenault E, Bonn F. Evaluation of soil erosion protective cover by crop residues using vegetation indices and spectral mixture analysis of multispectral and hyperspectral data. Catena, 2005, 62(2-3): 157-172.

[4]Purevdorj T S, Tateishi R, Ishiyama T, Honda Y. Relationships between percent vegetation cover and vegetation indices. International Journal of Remote Sensing, 2013, 19(18): 3519-3535

[5]Guerschman J P, Hill M J, Renzullo L J, Barrett D J, Marks A S, Botha E J. Estimating fractional cover of photosynthetic vegetation, non-photosynthetic vegetation and bare soil in the Australian tropical savanna region up scaling the EO-1Hyperion and MODIS sensors. Remote Sensing of Environment,2009, 113(5): 928-945.

[6]Somers B, Asner G P, Tits L, et al. Endmember variability in spectral mixture analysis: A review[J]. Remote Sensing of Environment, 2011, 115(7): 1603-1616.

[7]Asner G P, Bustamante M, Townsend A R. Scale dependence of biophysical structure in deforested areas bordering the Tapajos National Forest, Central Amazon[J]. Remote Sensing of Environment, 2003, 87(4): 507-520.

[8]Asner G P, Lobell D B. A biogeophysical approach for automated SWIR unmixing of soils and vegetation[J]. Remote sensing of environment, 2000, 74(1): 99-112.

[9]Zhu J F, Xing B L, Ju W M, Zhu G L, Liu Y B. Remote-sensing estimation of grassland vegetation coverage in Inner Mongolia, China. Chinese Journal of Plant Ecology, 2011, 35(6): 615-622.

[10]Zhang Wenting,Jin Keyi,Song Kaishan,et al.Imperious Surface Dynamic Quantification based on Multiple endmember Spectral Mixture Analysis and Landsat Imagery Data: A Case Study in Beijing[J]. Remote Sensing Technology and Application,2015,30(2):321-330

[11]Serbin G, Hunt E R, Daughtry C S T, McCarty G W, Doraiswamy P C. An improved SATER index for remote sensing of crop residue. Remote Sensing,2009, 1(4): 971-991.

[12]Yue Y M, Zhang B, Wang K L, et al. Spectral indices for estimating ecological indicators of karst rocky desertification. International Journal of Remote Sensing, 2010, 31 ( 8 ) :2115-2122. 